POS PROCEEDINGS

\title{
Upgrade of the GERDA Experiment
}

M. Agostini ${ }^{n}$, M. Allardt ${ }^{c}$, A.M. Bakalyarov ${ }^{l}$, M. Balata ${ }^{a}$, I. Barabanov ${ }^{j}$, N. Barros ${ }^{c}$, L. Baudis ${ }^{r}$, C. Bauer ${ }^{f}$, N. Becerici-Schmidt ${ }^{m}$, E. Bellotti ${ }^{g, h}$, S. Belogurov ${ }^{k, j}$, S.T. Belyaev ${ }^{l}$, G. Benato ${ }^{r}$, A. Bettini ${ }^{o, p}$, L. Bezrukov ${ }^{j}$, T. Bode ${ }^{n}$, D. Borowicz ${ }^{b}$, V. Brudanin ${ }^{d}$, R. Brugnera ${ }^{o, p}$, D. Budjás ${ }^{n}$, A. Caldwell ${ }^{m}$, C. Cattadori ${ }^{h}$, A. Chernogorov $^{k}$, E.V. Demidova ${ }^{k}$, A. Domula ${ }^{c}$, V. Egorov ${ }^{d}$, R. Falkenstein ${ }^{q}$, K. Freund ${ }^{q}$, N. Frodyma ${ }^{b}$, A. Gangapshev ${ }^{j, f}$, A. Garfagnini ${ }^{o, p}$, C. Gotti ${ }^{g, h}$, P. Grabmayr ${ }^{q}$, V. Gurentsov ${ }^{j}$, K. Gusev ${ }^{l, d, n}$, W. Hampel ${ }^{f}$, A. Hegai ${ }^{q}$, M. Heisel ${ }^{f}$, S. Hemmer ${ }^{o, p}$, G. Heusser ${ }^{f}$, W. Hofmann ${ }^{f}$, M. Hult ${ }^{e}$, L.V. Inzhechik ${ }^{j}$, L. loannucci ${ }^{a}$, J. Janicskó Csáthy $^{n}$, J. Jochum ${ }^{q}$, M. Junker ${ }^{a}$, V. Kazalov ${ }^{j}$, T. Kihm ${ }^{f}$, I.V. Kirpichnikov ${ }^{k}$, A. Kirsch ${ }^{f}$, A. Klimenko ${ }^{f, d}$, K.T. Knöpfle ${ }^{* f}$, O. Kochetov ${ }^{d}$, V.N. Kornoukhov ${ }^{k, j}$, V.V. Kuzminov ${ }^{j}$, M. Laubenstein ${ }^{a}$, A. Lazzaro ${ }^{n}$, V.I. Lebedev ${ }^{l}$, B. Lehnert ${ }^{c}$, H.Y. Liao ${ }^{m}$, M. Lindner ${ }^{f}$, I. Lippi ${ }^{p}$, A. Lubashevskiy ${ }^{f}$, B. Lubsandorzhiev ${ }^{j}$, G. Lutter ${ }^{e}$, C. Macolino ${ }^{a}$, B. Majorovits ${ }^{m}$, W. Maneschg ${ }^{f}$, G. Marissens ${ }^{e}$, M. Misiaszek ${ }^{b}$, I. Nemchenok ${ }^{d}$, S. Nisi ${ }^{a}$, D. Palioselitis ${ }^{m}$, L. Pandola ${ }^{a}$, K. Pelczar $^{b}$, G. Pessina ${ }^{g, h}$, A. Pullia $^{i}$, S. Riboldi ${ }^{i}$, N. Rumyantseva ${ }^{d}$, C. Sada ${ }^{o, p}$, M. Salathe $^{f}$, C. Schmitt ${ }^{q}$, J. Schreiner $^{f}$, O. Schulz ${ }^{m}$, B. Schwingenheuer ${ }^{f}$, S. Schönert ${ }^{n}$, E. Shevchik $^{d}$, M. Shirchenko ${ }^{l, d}$, H. Simgen ${ }^{f}$, A. Smolnikov ${ }^{f}$, L. Stanco ${ }^{p}$, H. Strecker ${ }^{f}$, C.A. Ur ${ }^{p}$, A.A. Vasenko ${ }^{k}$, K. von Sturm ${ }^{o, p}$, V. Wagner ${ }^{f}$, M. Walter ${ }^{r}$, A. Wegmann ${ }^{f}$, T. Wester ${ }^{c}$, H. Wilsenach ${ }^{c}$, M. Wojcik ${ }^{b}$, E. Yanovich ${ }^{j}$, P. Zavarise ${ }^{a}$, I. Zhitnikov ${ }^{d}$, S.V. Zhukov ${ }^{l}$, D. Zinatulina ${ }^{d}$, K. Zuber $^{c}$, and G. Zuzel ${ }^{b}$.

a) INFN Laboratori Nazionali del Gran Sasso, LNGS, Assergi, Italy

$\left.{ }^{b}\right)$ Institute of Physics, Jagiellonian University, Cracow, Poland

c) Institut für Kern- und Teilchenphysik, Technische Universität Dresden, Dresden, Germany

d) Joint Institute for Nuclear Research, Dubna, Russia

e) Institute for Reference Materials and Measurements, Geel, Belgium

f) Max-Planck-Institut für Kernphysik, Heidelberg, Germany

(list of institutes continued on next page)

The GERDA experiment searches for the neutrinoless double beta decay of ${ }^{76} \mathrm{Ge}$. In Phase I it has achieved an unprecedented background index of $10^{-2} \mathrm{cts} /(\mathrm{kev} \cdot \mathrm{kg} \cdot \mathrm{yr})$ at the $\mathrm{Q}$ value of the decay that allowed to set a new lower limit for the half-life of this lepton number violating process of $2.1 \cdot 10^{25} \mathrm{yr}(90 \%$ C.L.). This paper gives an overview of the present upgrade for Phase II where both background index and sensitivity shall be improved by about another order of magnitude.

Technology and Instrumentation in Particle Physics 2014,

2-6 June, 2014

Amsterdam, the Netherlands

*Speaker and corresponding author, E-mail: ktkno@mpi-hd.mpg.de. 
${ }^{g}$ ) Dipartimento di Fisica, Università Milano Bicocca, Milano, Italy

$\left.{ }^{h}\right)$ INFN Milano Bicocca, Milano, Italy

${ }^{i}$ ) Dipartimento di Fisica, Università degli Studi di Milano e INFN Milano, Milano, Italy

${ }^{j}$ ) Institute for Nuclear Research of the Russian Academy of Sciences, Moscow, Russia

$\left.{ }^{k}\right)$ Institute for Theoretical and Experimental Physics, Moscow, Russia

$\left.{ }^{l}\right)$ National Research Centre "Kurchatov Institute", Moscow, Russia

${ }^{m}$ ) Max-Planck-Institut für Physik, München, Germany

${ }^{n}$ ) Physik Department \& Excellence Cluster Universe, Technische Universität München, Germany

${ }^{\circ}$ ) Dipartimento di Fisica e Astronomia dell'Università di Padova, Padova, Italy

$p$ ) INFN Padova, Padova, Italy

${ }^{q}$ ) Physikalisches Institut, Eberhard Karls Universität Tübingen, Tübingen, Germany

$r$ ) Physik Institut der Universität Zürich, Zürich, Switzerland

\section{Introduction}

The GERmanium Detector Array (GERDA) experiment at the INFN deep-underground Laboratori Nazionali del Gran Sasso, Italy, has been designed for a most sensitive search of neutrinoless double beta $(0 v \beta \beta)$ decay of ${ }^{76} \mathrm{Ge},{ }^{76} \mathrm{Ge} \rightarrow{ }^{76} \mathrm{Se}+2 \mathrm{e}^{-}$, where the nucleus ${ }^{76} \mathrm{Ge}$ decays into ${ }^{76} \mathrm{Se}$ and two electrons. High-purity germanium diodes enriched in ${ }^{76} \mathrm{Ge}\left({ }^{e n r} \mathrm{Ge}\right)$ serve both as source and detector. The signature of the $0 v \beta \beta$ process is a peak at the transition energy $\mathrm{Q}_{\beta \beta}=2039 \mathrm{keV}$ in the electron sum spectrum, and its observation would indicate lepton number violation and the neutrino to be its own antiparticle, i.e. a Majorana fermion. If the decay is mediated by light neutrino exchange, it can be depicted as a standard $2 v \beta \beta$ decay $(\mathrm{A}, \mathrm{Z}) \rightarrow(\mathrm{A}, \mathrm{Z}+2)+2 \mathrm{e}^{-}+2 \bar{v}$ where the antineutrino of the one beta decay is absorbed as neutrino in an inverse beta decay process; it would carry then also information about the neutrino mass scale.

GERDA has used in Phase I $14.6 \mathrm{~kg}$ of refurbished semi-coaxial Ge detectors from the earlier IGEX and HDM experiments and $3.0 \mathrm{~kg}$ of Broad Energy Ge (BEGe) detectors (see section 3.1) all enriched to $\geq 86 \%$ in ${ }^{76} \mathrm{Ge}$ - and achieved for the exposure of $21.6 \mathrm{~kg} \cdot \mathrm{yr}$ the background index (BI) of $\sim 1 \cdot 10^{-2} \mathrm{cts} /(\mathrm{kev} \cdot \mathrm{kg} \cdot \mathrm{yr})$ that is a factor of 10 lower than in previous experiments. This progress allowed to set a new lower limit of $2.1 \cdot 10^{25} \mathrm{yr}$ at $90 \%$ C.L. ${ }^{1}$ for the half-life of $0 \mathrm{v} \beta \beta$ decay in ${ }^{76} \mathrm{Ge}$ [1], and to exclude a claim of observation [2] with $99 \%$ probability (Fig. 1, right).

In Phase II, GERDA is aiming to improve the half-life sensitivity by about one order of magnitude to $\sim 1.5 \cdot 10^{26} \mathrm{yr}$. At the end of Phase I GERDA has left, however, the zero background regime, $(\mathrm{M} \cdot \mathrm{t}) \times \mathrm{BI} \times \Delta \mathrm{E}<1,{ }^{2}$ where the sensitivity scales linearly with the exposure, $(M \cdot t)$, and entered the background i.e. statistical fluctuation limited scenario where it scales approximately with the square root of the exposure divided by BI times the energy resolution $\triangle \mathrm{E}$ (with the detection efficiency $\varepsilon$ included): $\varepsilon \sqrt{(M \cdot t) /(B I \cdot \Delta E)}$ (Fig. 1, right). A prolific upgrade requires thus to increase not only exposure (detector mass) but also to reduce accordingly background (BI) to re-enter the 'zero background' regime. Improvements in resolution $\Delta E$ are possible but limited to $\sim 45 \%$ for the given technology. Hence GERDA has to strive for a further significant reduction of the BI to $10^{-3} \mathrm{cts} /(\mathrm{keV} \cdot \mathrm{kg} \cdot \mathrm{yr})$ in order to reach the desired sensitivity beyond $10^{26} \mathrm{yr}$ at an exposure of about

\footnotetext{
${ }^{1}$ The median sensitivity of Phase I is $2.4 \cdot 10^{25} \mathrm{yr}$.

${ }^{2}(\mathrm{M} \cdot \mathrm{t}) \times \mathrm{BI} \times \Delta \mathrm{E} \sim 21.6 \mathrm{~kg} \mathrm{yr} \times 10^{-2} \mathrm{cts} /(\mathrm{keV} \mathrm{kg} \mathrm{yr}) \times 4.5 \mathrm{keV} \sim 1 \mathrm{cts}$
} 
$100 \mathrm{~kg} \cdot \mathrm{yr}$, or 2.5 life-years if the mass of deployed ${ }^{e n r} \mathrm{Ge}$ detectors would be doubled (Fig. 1, right). The measures taken to reach these goals are discussed in more detail below following a section with a short description of the overall GERDA setup. They include a further minimization of background sources in the array surrounding, as well as further developed front end electronics for better energy resolution. A major BI reduction will come from a largely improved discrimination of $0 v \beta \beta$ and background events by their different topology. While the former normally deposit energy at only one location in the diode, the latter will deposit energy also in the liquid argon (LAr) around the detectors or scatter at several locations in the detector. Background events can thus be identified (and vetoed) by their scintillation light in the LAr, by coincidences in granular/segmented detectors and/or by the analysis of the detector's pulse shape. GERDA will exploit all options in Phase II: the additional ${ }^{\text {enr }} \mathrm{Ge}$ diodes are of a novel type exhibiting pulse shape discrimination (PSD) of superior efficiency and energy resolution, a more densely packed detector array will exhibit an enhanced efficiency for detector-detector coincidences, and significantly, the LAr around the detector array will be instrumentend for the readout of scintillation light providing so an active LAr veto system,

\section{The GERDA experiment}

The GERDA experiment (Fig. 1, left) realizes a novel shielding concept by operating bare Ge diodes, enriched in ${ }^{76} \mathrm{Ge}$, in a large volume $\left(64 \mathrm{~m}^{3}\right)$ of high purity (5.0) LAr which serves both as cooling and shielding medium. The LAr is contained in a vacuum-insulated and actively cooled cryostat of $4.2 \mathrm{~m}$ diameter with internal copper lining; the cryostat itself is encased by a water tank of $10 \mathrm{~m}$ diameter. The highly purified water complements the shielding against the radiation from the surrounding rock and concrete; it serves also as a neutron shield, and instrumented with 66 photomultipliers tubes (PMTs) as Cherenkov medium for vetoing cosmic muons. The Ge detectors are deployed in strings from top into the cryostat using a glove box with dry nitrogen atmosphere for assembly, and a twin lock and suspension system for the deployment of a single resp. triple string. About $30 \mathrm{~cm}$ above the detector array, the signals of the Ge diodes are amplified by cryogenic low-
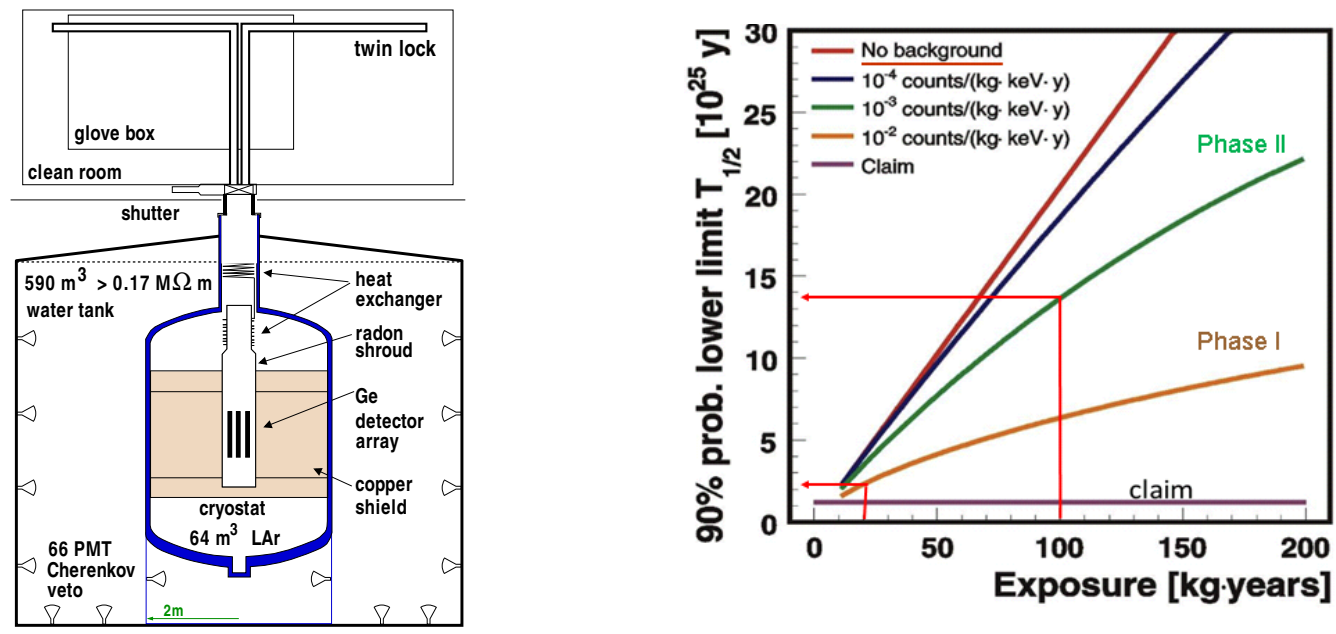

Figure 1: Left: GERDA schematic. Right: GERDA sensitivities in Phase I and II as well as the claim [2]. 
noise charge sensitive JFET-CMOS preamplifiers, and led via $\sim 10 \mathrm{~m}$ long coaxial cables to the outside of cryostat and lock for digitization with a 14 bit $100 \mathrm{MHz}$ flash ADC system. More details of the GERDA setup and the detector performance in Phase I are given in ref. [3].

\section{Upgrade to Phase II}

Fig. 2 shows a preview of the Phase II experimental setup. The size of the detector array is increased to seven strings which carry besides the Phase I semi-coaxial diodes additional $20 \mathrm{~kg}$ of novel enriched BEGe detectors with significantly improved performance. The vertical part of the
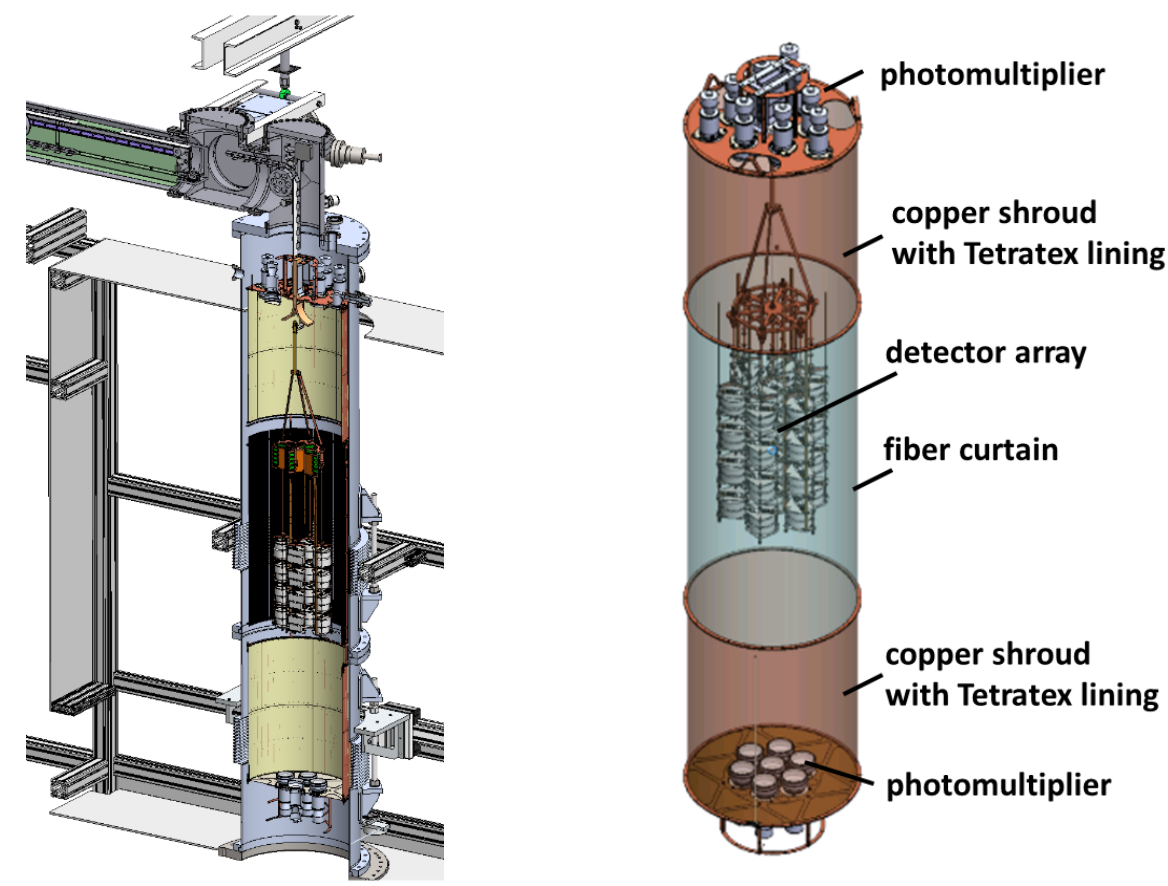

Figure 2: Left: Experimental arrangement for Phase II showing the detector array and LAr veto system within the new lock and glove box. Right: Close-up of the assembly of detector array and surrounding LAr veto system as it will be immersed into the cryostat.

new lock with increased diameter and height is enclosed by the glove box. The design allows to assemble both the detector array and the surrounding LAr veto system in the open lock under dry nitrogen atmosphere, and to lower both systems together into the cryostat.

\subsection{The new batch of enriched BEGe detectors}

Although discrimination of localized and multiple energy deposits by PSD has been performed successfully with the semi-coaxial Ge diodes in Phase I [4], GERDA has studied for Phase II other detector designs which warrant more efficient PSD due to a more favourable electric field distribution and/or electrode segmentation. The original candidate for Phase II, a true coaxial n-type detector with segmented electrodes, showed indeed the expected improved performance [5]. It was discarded, however, in part because of the difficulty to procure n-type crystals from enriched material but also because a commercially available point contact detector type [6] built with p-type 
material, the BEGe detector, had been identified exhibiting at least similar PSD performance, and in addition better energy resolution due to a very low detector capacitance [7].

The basic raw material for the production of the new batch of BEGe diodes enriched in ${ }^{76} \mathrm{Ge}$ has been procured in form of $53.4 \mathrm{~kg}$ of ${ }^{e n r} \mathrm{GeO}_{2}$ from ECP, Zelonogorsk, Russia, with the ${ }^{76} \mathrm{Ge}$ isotope enriched to $\sim 88 \%$; a similar amount of simultaneously produced depleted material ${ }^{d e p l} \mathrm{GeO}_{2}$ was used to validate the complete production chain [9]. The reduction and purification of the $\mathrm{GeO}_{2}$ was achieved with an efficiency of $94 \%$ yielding $35.5 \mathrm{~kg}{ }^{\mathrm{enr}} \mathrm{Ge}(6 \mathrm{~N})$ for crystal production at Canberra, Oak Ridge. Adopting the optimized production scheme from the ${ }^{d e p l} \mathrm{Ge}$ test run, a total of 9 crystals could be pulled. The crystals were cut into 30 slices and sent to Canberra, Olen, where they were transformed into working BEGe detectors with a total mass of $20.0 \mathrm{~kg}$. All detectors have
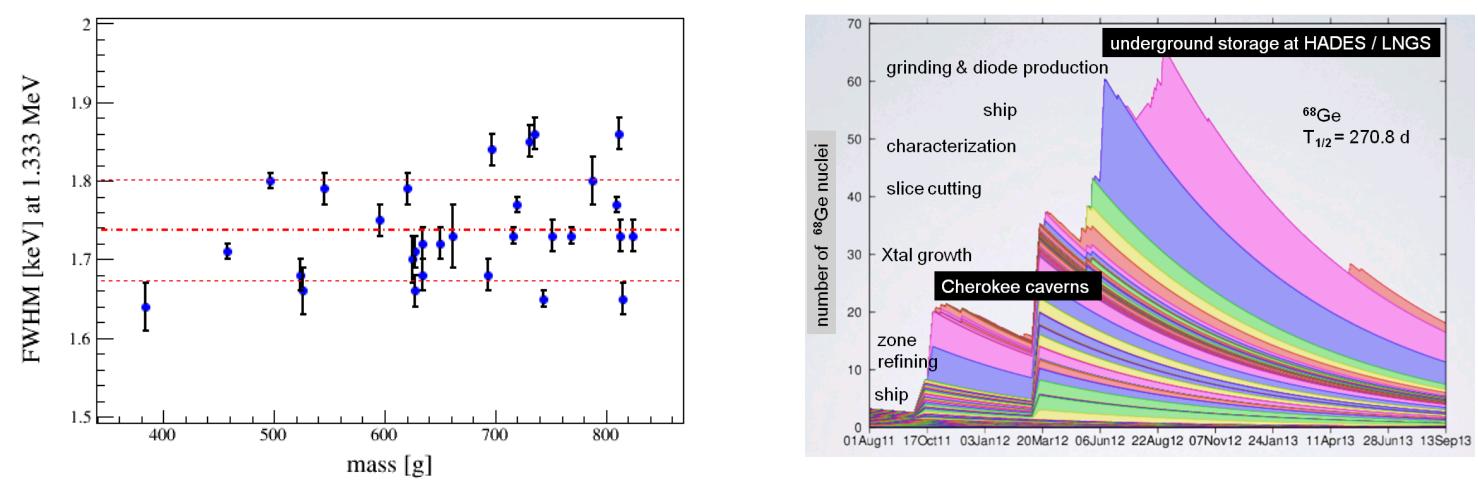

Figure 3: Left: Mass and FWHM resolution at $1.3 \mathrm{MeV}$ of the new BEGe detectors. Right: Online log of the activation of a specific BEGe diode by ${ }^{68} \mathrm{Ge}$ that has been cosmogenically created during indicated production steps; black labels denote periods of (partly interrupted) underground storage at indicated locations.

been characterized in the HADES underground facility close to Olen with respect to operational parameters including active volumes, dead layers and pulse shape performance; 29 work according to specifications reaching full depletion with bias voltages below $5 \mathrm{kV}$ and an energy resolution at $1.3 \mathrm{MeV}$ of $<1.9 \mathrm{keV}$ (FWHM); their average mass is $(667 \pm 115) \mathrm{g}$ (Fig. 3, left). In a final step, $\mathrm{Al}$ contacts for wire bonding were evaporated on the $\mathrm{p}^{+}$and $\mathrm{n}^{+}$substrates of each crystal, and the passivation layer in the groove was removed. During all production steps, the exposure of the enriched material to the cosmic radiation has been reduced significantly by shielded transport and/or underground storage of the material (Fig. 3, right); hence its cosmic activation could be limited to less than twenty-two ${ }^{60} \mathrm{Co}$ and less than five ${ }^{68} \mathrm{Ge}$ nuclei per kg contributing after PSD to the BI $<2 \cdot 10^{-6} \mathrm{cts} /(\mathrm{keV} \cdot \mathrm{kg} \cdot \mathrm{yr}) \mathrm{resp} .<9 \cdot 10^{-6} \mathrm{cts} /(\mathrm{keV} \cdot \mathrm{kg} \cdot \mathrm{yr})$.

\subsection{Lock}

The new Phase II lock has evolved from the proven design of the Phase I lock [3] by merging the two cable arms to a single one and enlarging the lock diameter to $500 \mathrm{~mm}$. This allows to deploy a closely packed detector array of at least seven detector strings together with the complete assembly of the LAr instrumentation (Fig. 2). The detector array will consist of four strings, each with four pairs of BEGe diodes, and three strings with up to 9 Phase I semi-coaxial detectors. The new cable chain is made of selected stainless steel of low radioactivity and specified for a load of 
up to $60 \mathrm{~kg}$. Its increased cross section of $2 \times 10 \mathrm{~cm}^{2}$ holds $70 \mathrm{RG} 179$ high voltage cables, $12275 \Omega$ and 48 RG178 $50 \Omega$ signal cables, all woven in five cable ribbons. These coaxial cables, being all custom produced with uncoloured PFA as dielectric and jacket, exhibit specific ${ }^{228} \mathrm{Th}$ and ${ }^{226} \mathrm{Ra}$ activities that are more than factor of 10 lower than the Phase I cables.

\subsection{The BEGe detector module}

Fig. 4 shows a Phase II BEGe detector module, four of which will be assembled to form one string. It consists of 2 BEGe diodes which are mounted back-to-back. Since the evaluation
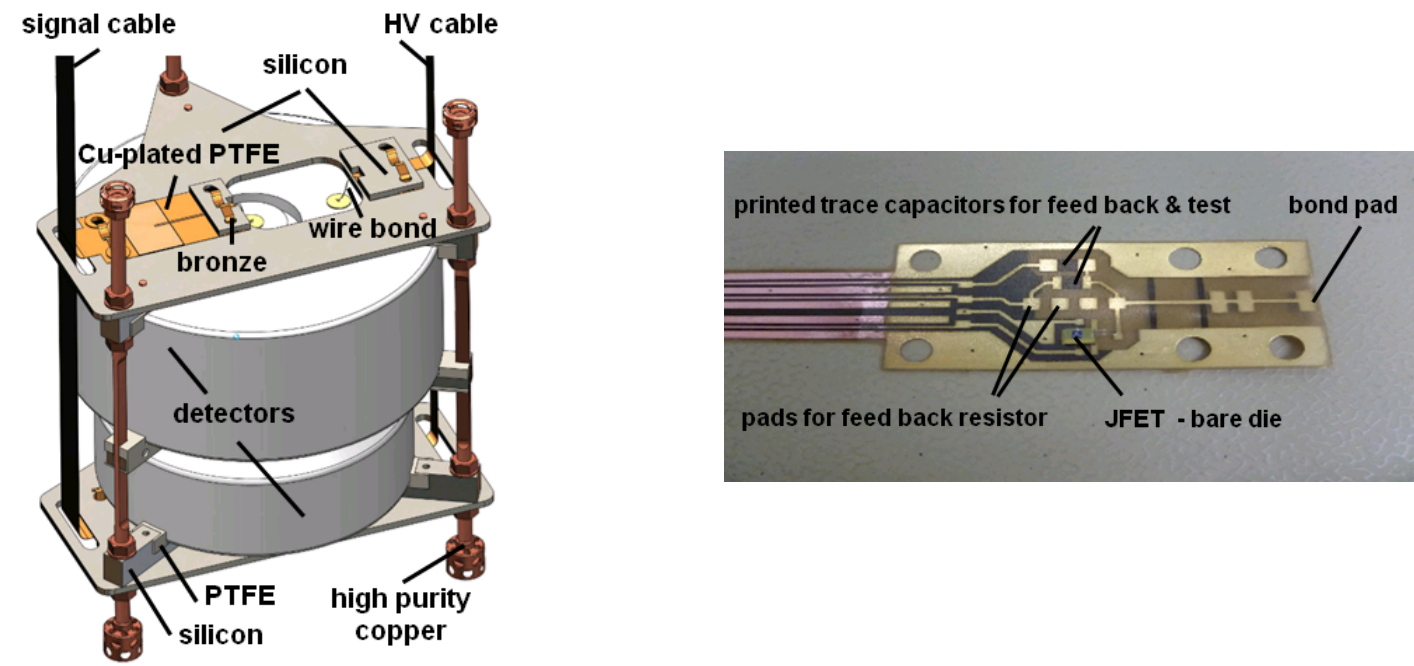

Figure 4: Left: Phase II detector module of a BEGe detector pair in low-mass mount, Right: Flexcable for signal $(\mathrm{p}+)$ contact carrying JFET and custom-made feed back resistor.

of the GERDA Phase I background has shown [8] large part of the background to originate from sources close to the Ge detectors, like detector supports, mini-shrouds or cables, the design is optimized such that both the amount of surrounding material is further reduced and/or the material itself is replaced by another kind of material of higher radio-purity. The new design replaces the Phase I spring-loaded contacts to the detectors by wire bonds. Thus one third of the copper (and PTFE) per detector mass unit could be replaced by mono-crystalline silicon which is less strong but intrinsically radio-pure. An equivalent design will be also used for the semi-coaxial diodes. The module warrants also the fixation of the flexible bias and signal cables for the BEGe pair which are custom made from CuFlon [12]. The signal cables lead to low-noise cryogenic preamplifiers which are placed about $50 \mathrm{~cm}$ above the top of the array. To take full advantage of the low input capacity of the BEGe diodes, the very front end is - other than in Phase I - moved very close to the detector where the cable carries a layout (Fig. 4, right) for the placement and wire bonding of the JFET, a commercial bare die (SF291), a custom-made feed back resistor ( $\sim 1 \mathrm{G} \Omega$ ), and a printed trace feed back capacitor $(0.3 \mathrm{pF})$. The preamplifiers are an upgrade of the charge sensitive device $\mathrm{CC} 2$ [3] of Phase I based on commercially available components. They feature $2.6 \mathrm{keV}$ FWHM at $2.6 \mathrm{MeV}$ with a BEGe detector, $20 \mathrm{MHz}$ band width allowing PSD with the A/E method [4], 50mW/channel power dissipation that is suitable for operation in LAr, and a radio-purity of $50 \mu \mathrm{Bq} /$ channel including connectors. 


\subsection{LAr instrumentation and transparent mini-shrouds}

The LAr veto system is a hybrid system (Fig. 2, right) that has evolved from studies of scintillation light detection in LAr with 8" PMTs in the LArGe test stand [10] and silicon photomultipliers (SiPMs) coupled to wavelength shifting fibers for increasing the light detection efficiency [11]. In GERDA a cylindrical volume of $220 \mathrm{~cm}$ height and $49 \mathrm{~cm}$ diameter with the detector array in the center, will be watched from top and bottom by 9 resp. 7 PMTs (Hamamatsu, 3" R11065-10/20 MOD) of low radioactivity $(<2 \mathrm{mBq} / \mathrm{PMT})$ and low power $(17 \mu \mathrm{A}$ at $1.5 \mathrm{kV})$. While the upper and lower parts of this volume, $60 \mathrm{~cm}$ in height each, consist of thin-walled $(0.1 \mathrm{~mm})$ copper cylinders lined by wavelength shifting reflector foils (Tetratex + TPB), the central part of $100 \mathrm{~cm}$ height features a 'curtain' made of $1 \times 1 \mathrm{~mm}^{2}$ wavelength shifting fibers coated with TPB and coupled in groups of nine to 3x3 SiPM arrays (Ketek) (Fig. 5, left). Pointing with their diagonals to the central axis, the total of 810 fibers covers almost $80 \%$ of the circumference. In particular, they collect the
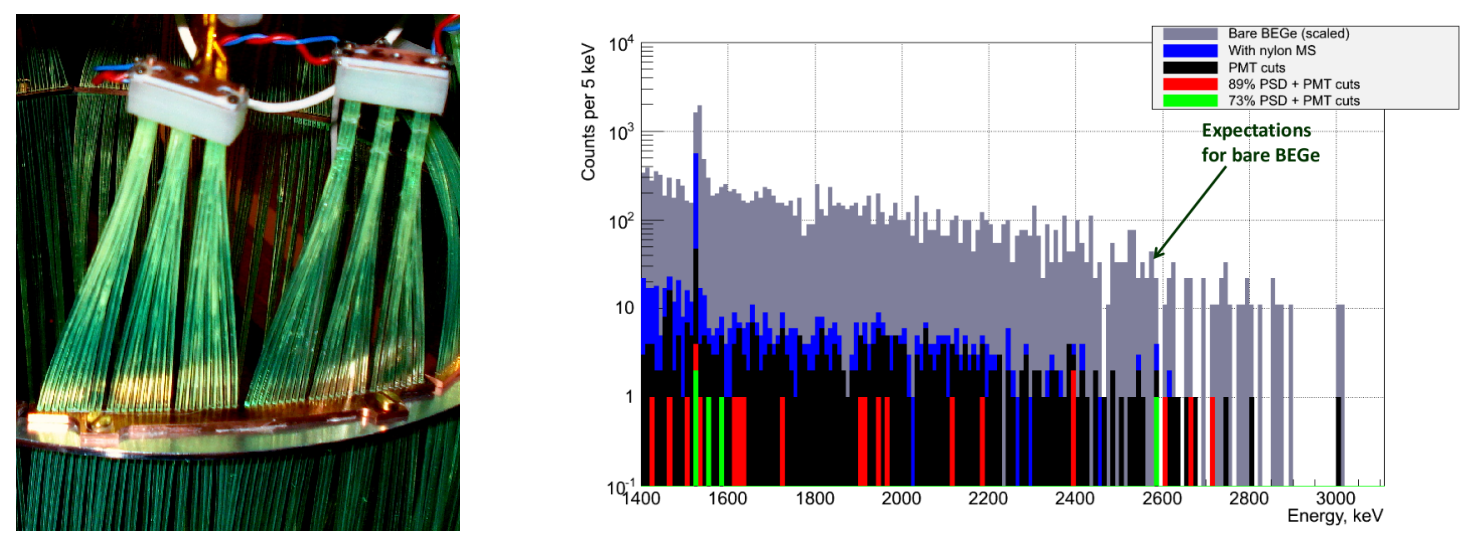

Figure 5: Left: Detail of the top part of the fiber curtain showing fiber holders, the grouping of fibers into the optical couplers, and on top of them the SiPMs. Right: Spectrum of BEGe detector measured in the test stand spiked with ${ }^{42} \mathrm{Ar}$, and corresponding spectra obtained with the nylon mini-shroud and indicated cuts.

light of scintillating events from both the inside and outside of the shroud. Being deployed together with the detector array, this setup exhibits a large LAr volume and minimizes shadowing effects by the detector array. Detailed Monte Carlo simulations with tracking of XUV and optical photons predict background reduction factors of 3 to 10 for ${ }^{214} \mathrm{Bi}$ depending on its location, and of 60 to 300 for ${ }^{208} \mathrm{Tl}$ located close to the detector array.

In Phase I almost hermetically closed copper mini-shrouds were essential for reaching the desired BI; providing both a barrier against convection and a shield for the electric fields of the biased detectors, they reduced in a small volume around each detector string the contamination by ${ }^{42} \mathrm{~K}$ ions $\left(\mathrm{T}_{1 / 2}=12.4 \mathrm{~h}, \mathrm{E}_{\beta}=3.5 \mathrm{MeV}\right)$, progenies of ${ }^{42} \mathrm{Ar}$ decays [3]. Given the LAr instrumentation of Phase II, a transparent or optically active mini-shroud is required to detect also the light emitted by backgrounds such as ${ }^{214} \mathrm{Bi}$ close to the detectors. Several options including a biased copper mesh and a CuFlon shroud with an internal large-area SiPM have been successfully tested in the LArGe test stand which had been spiked with artificially produced ${ }^{42} \mathrm{Ar}$ for increased statistics. The chosen option with best performance is a hermetically closed mini-shroud made from transparent nylon and coated with TPB as wave length shifter (Fig. 5, right). 


\section{Status and Conclusion}

GERDA Phase I has been concluded in September 2013. After more than 3 years of operation the water tank was emptied. Selected welds and surfaces of both cryostat and water tank were inspected by a representative of a notified body. No corrosion problems were observed and the system safety of the pressure equipment certified. The access to the water tank allowed also to replace two of the three broken (imploded, in fact) PMTs of the Cherenkov system. With the Phase I lock dismantled, the $20 \mathrm{kBq}{ }^{228} \mathrm{Th}$ calibration source, dropped during Phase I by accident to the bottom of the cryostat, was rescued without emptying the cryostat. While it did not contribute significantly to the BI of Phase I [8] its presence would be not tolerable in Phase II.

At the date of writing, June 2014, the new lock is fully operational, and the commissioning of the Phase II detector assembly will start with the deployment of a BEGe pilot string. The successful implementation of the Phase II hardware met various challenges the majority of which are due to the severe constraint for the radio-purity of the individual components which is verified by advanced screening methods. This issue prevents standard solutions and requires in many instances the custom production of components including ${ }^{e n r} \mathrm{Ge}$ detectors, cables, resistors, and even of construction materials like ultra-pure copper, bronze or PTFE. Similarly, a collaboration with the supplier of PMTs of low-radioactivity for cryogenic operation was crucial in order to solve the problem of unexpected flashing.

The operation of a large Ge detector array together with the active LAr veto system will help to identify the nature and origin of the relevant background components which could not be extracted unambiguously from the Phase I data. With such information the algorithms and cuts for PSD are expected to be further improved. This will support the Phase II hardware upgrade for reaching the goal of a half-life sensitivity beyond $10^{26} \mathrm{yr}$ for $0 v \beta \beta$ decays of ${ }^{76} \mathrm{Ge}$. Eventually, the experience of Phase II might be helpful for understanding if the technology is suitable for a ton scale experiment.

\section{References}

[1] The Gerda Collaboration, M. Agostini et al., Phys. Rev. Lett. 111 (2013) 122503.

[2] H.V. Klapdor-Kleingrothaus et al., Phys. Lett. $\mathbf{B 5 8 6}$ (2004) 198.

[3] The Gerda Collaboration, K.-H. Ackermann et al., EPJC 73 (2013) 2330.

[4] The Gerda Collaboration, M. Agostini et al., EPJC 73 (2013) 2583.

[5] I. Abt et al., Nucl. Instr. Methods A583 (2007) 332-340.

[6] P.N. Luke et al., IEEE Trans. Nucl. Sci. 36 (1989) 926; P.S. Barbeau et al., JCAP 09 (2007) 009.

[7] D. Budjáš et al., JINST 4 (2009) P10007.

[8] The Gerda Collaboration, M. Agostini et al., EPJC 74 (2014) 2764.

[9] D. Budjáš et al., JINST 8 (2013) P104018.

[10] M. Agostini et al., J. Phys.: Conf. Ser. 375 (2012) 042009.

[11] J. Janicskó Csáthy et al., Nucl. Instr. Methods 654 (2011) 225-232.

[12] Registered trademark of Polyflon Company, http://www.polyflon.com/microw.htm . 